and that each of these peripheral fibers, in its turn, is divided into two. These relationships may be indicated diagrammatically. One pyramidal fiber may, therefore, be represented

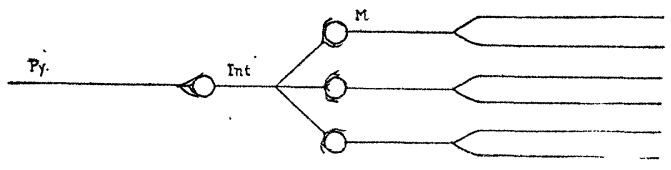

FIG. 1.

at the periphery by six branches of axones, each of which is in its turn capable of acting upon an effector. The energy, $a$, coming down the first fiber in the series, $P y$, is, according to the all or none hypothesis, the maximum which the fiber is capable of conducting. Similarly, the energy passing over the intercalated (Int.) fiber before its branching is also the maximum which it is capable of conducting. Suppose that it is equal to $a$. At the point of branching, the energy conducted along each branch must either be brought up to some quantity closely approximating $a$, or else it must fall to $a / 3$. In the latter case, the energy passing over the proximal unbranched portion of the fiber $M$ must either be brought up to the value $a$, or else in its turn be close to the value $a / 3$. Going on out to the bifurcation of this fiber, there must again be a raising of the energy in each of the branches to some value closely approximating $a$, or else it must fall to a value $a / 6$. There is little or no evidence that the energy of the nerve impulse falls off in any such degree in its passage from central system to periphery. The presumption is, therefore, that the efferent distribution path acts as a step-up transformer of energy, although the manner of its action is as yet unknown. It should be stated here that the nerve fiber itself furnishes the energy, derived in some manner as yet unknown from its own metabolic processes, and that there is, in all probability, no change in voltage at the expense of the amperage, as in the electrical transformers with which the physicist is familiar.
Reference to Ranson's ${ }^{1}$ diagram of the sympathetic system will show that the same considerations apply there. In fact, the diagram given in this paper was suggested by Ranson's diagrams.

One more link in the scheme of the step-up transformer may be what Langley has called the receptive substance, interposed, chemically if not histologically, between the motor end plates and the contractile substance in muscle. It is certain that there is a great increase in the energy of a muscle contraction as compared with the energy of the nerve impulse, which, starting in the central system, finally evokes the muscle contraction at the periphery. It seems reasonable to suppose, in the light of our present knowledge, that the efferent nerve path is a part of this transformer system.

Such general relationships of the energy of the response to the energy changes in the processes preceding the response have long been recognized. Balfour Stewart ${ }^{2}$ (p. 163) remarks: "We have seen that life is associated with delicately-constructed machines, so that whenever a transmutation of energy is brought about by a living being, could we trace the event back, we should find that the physical antecedent was probably a much less transmutation, while again the antecedent of this would probably be found still less, and so on, as far as we could trace it." We should recognize, however, that such relationships have a limit in the living organism. Otherwise, we would arrive at perpetual motion.

F. H. PIKE

Departinent of Phystology, Columbia UNIVERSITT

\section{ON SPIRAL NEBULAE}

ONE of the privileges of the vacation is the opportunity of making one's own tea in one's own vessels. I did so recently, aided by a deep precipitation glass, $g$, with a lip, $l$, running far down the sides. On stirring the

1 Ranson, S. W., 1918, Journal of Comparative Neurology, Vol. 29, p. 306.

2 Stewart, Balfour, 1874, "The Conservation of Energy,' New York, p. 163. 
liquid with a spoon, energetically, and removing the latter, I noticed that a sharply outlined spiral was persistently present on the surface, until the deep paraboloid returned to the plane. My explanation would be, that at $l$, part of the tangential velocity is converted into local vortical motion, whereby the particles at $l$, because of the reduced centrifugal force, slide down the inclined plane of the rotating paraboloid. From another point of view, a stationary wave is produced on the surface by the interference at $l$.

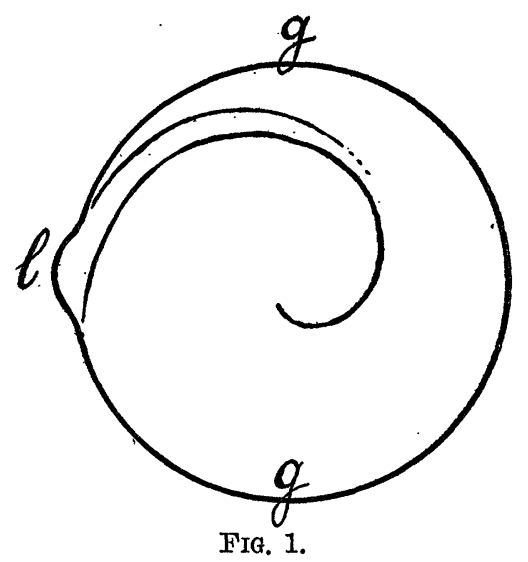

Now though I will not venture to repeat the superscript of this note, I will nevertheless ask whether something analogous to the above simple experiment may not be taking place in astronomical space. Suppose we replace the glass vessel of the figure by a gravitational mechanism; and suppose we "lip" it at $l$, by making that locality a region of effectively greater density and relatively at rest. If Kepler's law be written in the form so convenient in its present relations to the modern atom ( $M$, being the virtual mass at the center and $A$ the angular momentum per gram, whereby $r v^{2}=A^{2} / r=M$, for the tangential velocity $v$ at $r$ ), then any local diminution of $A$ in accordance with the above model, would be followed by a diminution of $r$ in the part affected.

At all events the hydrodynamic experiment (rotational surface figures, as related to shape of boundary) is very beautiful and certainly more approachable. I shall allow myself to play with more interpretable modifications of it a little longer.

BROWN UNIVERSITY

Carl Barus

\section{THE PACIFIC DIVISION OF THE AMERICAN AS- SOCIATION FOR THE ADVANCEMENT OF SCIENCE}

The Seattle meeting of the Pacific Division of the American Association for the Advancement of Science held. June 17 to 19 at the University of Washington, Seattle, was perhaps the most successful so far held by the Pacific Division. Sixteen affiliated societies were scheduled in the final program and delegates were in attendance from every part of the $\mathrm{Pa}$ cific Coast area. The representation from the University of California and Stanford Univensity was particularly large.

The special sessions of the convention in which the various affliated societies participated were well attended and the beneficial results of this cooperation were apparent. The conference of Research Committees from the educational institutions of the Pacific Coast held two sessions which were attended by all the delegates. The problems connected with the maintenance and encouragement of active research in the college and university were presented and discussed and some practical suggestions were made. It was felt that distinct progress in the solution of these problems had resulted from this meeting and that the research conference should be a permanent feature of the annual meetings of the Pacific Division.

A symposium on the "Einstein Theory of Relativity" was of general interest and was also well attended. In the symposium on "The Animal and Plant Resources of the North Pacific Ocean" given under the auspices of the Pacific Fisheries Society and the Western Society of Naturalists, each speaker emphasized the great need for more knowledge of the ocean and its life to save the fisheries industry. It is hoped that the means will be found to publish the papers in this symposium as a contribution to a better understanding of the importance of the projected exploration of the North Pacific Ocean through international co- 